DOI 10.35953/raca.v1i1.39

\title{
Entre olvidos, omisiones, colonialidades en lucha y paradigmas emancipatorios de los patrimonios alimentarios
}

Between forgetfulness, omissions, struggling colonialities and emancipatory paradigms of food heritage

Entre esquecimento, omissões, colonialidades em conflito e paradigmas emancipadores da herança alimentar

\section{Gloria Veronica Sammartino ${ }^{1}$}

${ }^{1}$ Doctora en Antropología Social, Investigadora. Escuela de Nutrición, Universidad de Buenos Aires. E-mail: gloriasammartino@gmail.com

\section{RESUMEN}

Desde el abordaje de una perspectiva decolonial el objetivo de este artículo es identificar el juego de visibilidades e invisibilidades implícitos en los procesos de patrimonialización y etnomercantilización, como las tensiones y alteridades emergentes en las prácticas alimentarias de sectores estigmatizados y negados del sur global. Se presentan tres casos que se desprenden de distintos estudios centrados en temáticas alimentarias. En el primero se destaca la emergencia de alimentos y comidas "rescatadas" para el mercado turístico que en su reestetización ocultan la otredad que remite a raíces indígenas y campesinas. En el segundo se observa la reconfiguración de identidades alimentarias a partir de la circulación de comidas de inmigrantes donde aparecen las marcas del racismo, la xenofobia y la reconfiguración mercantilizada de las mismas tras el pujante boom que asumen las comidas de esta comunidad a nivel internacional. En el tercero se destaca el "saber hacer" de grupos migrantes que en el nuevo contexto siguen incorporando los gustos y sabores en los que se reconocen como portadores e integrantes de una comunidad que no desata entusiasmo como recurso cultural por parte de la sociedad receptora. Los tres casos poseen elementos que encarnan y tensan categorizaciones raciales históricamente construidas y legitiman las relaciones de superioridad/inferioridad, reproduciendo legados coloniales que perpetúan la desigualdad social. Finalmente se busca mostrar conexiones entre patrimonios alimentarios emancipatorios y estrategias dentro del marco del derecho a la alimentación.

Palabras claves: Patrimonios alimentarios; Decolonización; Desigualdad; Mercado.

\section{ABSTRACT}

From the perspective of a decolonial perspective, the objective of this article is to identify the game of visibilities and invisibilities implicit in the processes of patrimonialization and ethnomercantilization, such as the emerging tensions and 


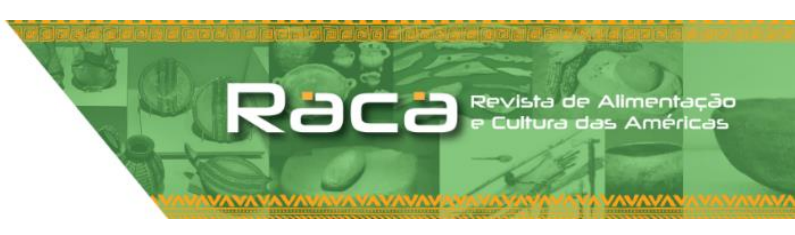

DOI 10.35953/raca.v1i1.39

alterities in the food practices of stigmatized and denied sectors of the global south. Three cases are presented that emerge from different studies focused on food issues. In the first, the emergency of food and meals "rescued" for the tourist market stands out, which in its re-aestheticization conceals the otherness that refers to indigenous and peasant roots. In the second, the reconfiguration of food identities is observed from the circulation of immigrant foods where the marks of racism, xenophobia and the commercialized reconfiguration of them appear after the vigorous boom that the meals of this community assume at the international level. In the third, the "know-how" of migrant groups stands out, which in the new context continue to incorporate the tastes and flavors in which they are recognized as carriers and members of a community that does not unleash enthusiasm as a cultural resource on the part of the receiving society. The three cases have elements that embody and tense historically constructed racial categorizations and legitimize superiority/inferiority relationships, reproducing colonial legacies that perpetuate social inequality. Finally, it seeks to show connections between emancipatory food heritage and strategies within the framework of the right to food.

Keywords: Food heritage; Decolonization; Inequality; Market.

\section{RESUMO}

Do ponto de vista de uma perspectiva descolonial, o objetivo deste artigo é identificar o jogo de visibilidades e invisibilidades implícitas nos processos de patrimonialização e etnomercantilização, como as tensões e alterações emergentes nas práticas alimentares de setores estigmatizados e negados do sul global. São apresentados três casos que emergem de diferentes estudos focados em questões alimentares. Na primeira, destaca-se a emergência de alimentos e refeições "resgatados" para o mercado turístico, que em sua reestetização oculta a alteridade que se refere às raízes indígenas e camponesas. No segundo, a reconfiguração das identidades alimentares é observada a partir da circulação de alimentos imigrantes, onde as marcas de racismo, xenofobia e a reconfiguração comercializada deles aparecem após o vigoroso boom que as refeições dessa comunidade assumem em nível internacional. No terceiro, destaca-se o "know-how" dos grupos de migrantes, que no novo contexto continuam a incorporar os gostos e sabores nos quais são reconhecidos como transportadores e membros de uma comunidade que não desencadeia o entusiasmo como recurso cultural por parte da sociedade receptora. Todos os três casos têm elementos que incorporam e pressionam categorizações raciais historicamente construídas e legitimam relações de superioridade/inferioridade, reproduzindo legados coloniais que perpetuam a desigualdade social.Por fim, busca mostrar conexões entre o patrimônio emancipatório e as estratégias alimentares no âmbito dos movimentos de direito à alimentação.

Palavras-chave: Herança alimentar, descolonização, desigualdade, mercado. 
DOI 10.35953/raca.v1i1.39

La cocina constituye uno de los modos por los cuales las identidades se materializan, se actualizan prácticas del pasado y se reintroducen como referencias contemporáneas, conectando y preservando los sabores, los modos de preparación, la memoria personal y colectiva. Permite expresar vínculos emocionales y afectivos que reafirman y refuerzan sentimientos de pertenencia, de diferenciación entre grupos, segmentos sociales, geografías, regiones y culturas. Sin embargo, estas prácticas y sentidos no son inmutables en el tiempo. Se modifican y resignifican en el marco de los contextos históricos y las dinámicas conformadas en la esfera internacional, tanto en términos de comercio como de movimientos poblacionales. Desde hace siglos las fuerzas del comercio y la conquista vienen transportando alimentos y plantas, convirtiendo espacios geográficos en plataformas exportadoras, haciendo que productos exóticos como el té, café, azúcar, chocolate se convirtieran en ingredientes masivos y pasaran a ser considerados tradicionales en países no productores ${ }^{(1)}$ (2).

A escala global, las transformaciones estructurales del sistema agroalimentario han generado nuevos productos y formas de producir, incidido en la incorporación de nuevas referencias de consumo y redefinido culturas y tradiciones en el interior de las sociedades locales. A mediados del siglo XX, a raíz de la internacionalización del capital industrial y de la consolidación del sistema agroalimentario actual, las corporaciones de la industria alimentaria se convierten en el centro de la pauta de consumo, creando nuevos productos, apropiándose de las tradiciones y redefiniéndolos sobre la base de nuevos conceptos comerciales ${ }^{(3)}$. Para lo cual destinan cifras multimillonarias a la publicidad y la propaganda, bajo la premisa de que los alimentos sean "buenos para vender" más allá de sus cualidades nutricionales ${ }^{(4)}$. Los negocios gastronómicos, como parte de estas tendencias, comenzaron a transportar y adaptar platos típicos, tras lo cual tanto el plato original se altera mediante la incorporación de nuevos ingredientes, mientras que los hábitos alimenticios locales se transforman. Así es como, por ejemplo, la cocina japonesa, tailandesa, mexicana, peruana y turca se ha convertido en la gastronomía local de muchos pueblos, en una diversificación globalizadora dirigida mucho más por factores económicos que por el desplazamiento de la población ${ }^{(1)}$. 
En este contexto, entre tendencias globalizadoras y gloacalizadoras, anclamos la relación entre la alimentación y la patrimonialización. Es de señalar que como campo de estudio desde la perspectiva antropológica es relativamente reciente. Emerge tras que la UNESCO ${ }^{(5)}$ señalara, hacia 1996, la inclusión de las artes culinarias como un campo del patrimonio inmaterial y un bien para la competitividad económica y turística. Entre los múltiples desarrollos que se han venido realizando nos interesan en particular los que identifican y analizan los mecanismos de adquisición de valor de la patrimonialización, de «lo auténtico» como clave para la mercantilización del patrimonio (6) (7) (8) (9). Desde esta perspectiva es posible dar cuenta de la construcción de una imagen de las identidades convertidas para el mercado en nuevos recursos mercantiles. Esto a través del tratamiento de la otredad presentada como atractiva, fetichizada y exaltada en su diversidad a través de nociones de tradición, antigüedad y autenticidad. También a través de recursos que apuntan a descontextualizar los alimentos, junto a un "saber hacer" de personas "distantes", que por diversas razones han sido estigmatizadas, negadas, y cuyas culturas e historias son asumidas como atraso en el marco de los impactos del proyecto civilizatorio eurocéntrico (10). La noción de etnomercancias (11) resulta interesante pues permite dar cuenta del papel que pueden jugar la cultura y la identidad de productores de alimentos "olvidados", asociados a pueblos indígenas, personas de color, migrantes y todos los pueblos marginados y oprimidos. Pues lo que se busca desde esta perspectiva es apelar y evocar a la noción del "otro" permitido" que ha pasado "el test de la modernidad" (12), a través de rasgos que evocan en el comensal su acercamiento a algo placentero y desconocido a la vez.

Otro mecanismo del que podríamos decir echa mano el mercado, es el carácter de «espectacular» con el que se construye el patrimonio alimentario (6). La espectacularización apunta a facilitar el consumo de bienes y servicios destinados al ocio, al tiempo libre y al turismo en general. Esto a través de la generación de un relato donde el comensal se integra en la comunidad emisora del patrimonio alimentario, pudiendo interiorizarse del mismo como una realidad auténtica y definidora de la identidad de aquellos que lo construyen y lo exponen, incluso cuando el producto tiene poco de auténtico en esencia. Es decir que los bienes considerados patrimonio y que se perciben como propios de una tradición local, más 
DOI 10.35953/raca.v1i1.39

allá de pretender simbolizar una identidad determinada, acaban siendo explotados en el mercado de consumo con la pretensión de fomentar el desarrollo territorial, social y económico (8).

En este escenario presento tres situaciones, una de ellas se desprende de mi tesis doctoral, centrada en el estudio de significados alimentarios de pobladores rurales de Quebrada de Humahuaca durante los años 2005 y 2015, en la que emergen alimentos y comidas "rescatadas" para el mercado turístico tras la patrimonialización por Unesco en $2004{ }^{(13)}$ pero que en su reestetización ocultan la otredad que remite a raíces indígenas y campesinas. La segunda se enmarca en el trabajo realizado para mi tesis de licenciatura, entre los años 1999 y 2001 (14) (15) centrada en la reconfiguración de las identidades a partir de la circulación de comidas de inmigrantes peruanos en el área metropolitana de Buenos Aires, donde aparecen las marcas del racismo y la xenofobia por parte de la sociedad receptora. El "regreso al campo" cerca de diez años después, permite ver la repercusión que adquiere en el mismo contexto el pujante boom de la comida peruana a nivel internacional y la reconfiguración "mercantilizada" de las identidades. La tercera situación se desprende de una investigación recientemente realizada, sobre prácticas y significados que enlazan la temática de la alimentación, la salud y la obesidad, entre pobladores urbanos de barrios populares del área metropolitana bonaerense, en muchos casos de origen paraguayo, y productores de verduras de zonas periurbanas bonaerense de origen boliviano. Del trabajo de campo de este estudio emerge que el "saber hacer" de estos "otros" que continúan en el nuevo contexto incorporando los gustos y sabores en los que se reconocen como portadores e integrantes de una comunidad no desata entusiasmo como recurso cultural por parte de la sociedad receptora.

De la presentación sucinta de estas tres situaciones es posible formular algunas preguntas: ¿Cuáles son las tensiones que expresa la patrimonialización?, ¿En qué marcas pueden verse la reproducción de las fuerzas y legados coloniales en la selección de lo "bueno para patrimonializar"?, ¿qué se ilumina y que se oculta en este proceso guiado por las lógicas del mercado? En el intento por esbozar algunas respuestas el objetivo de este artículo, desde el abordaje de una perspectiva decolonial, es identificar el juego de visibilidades e invisibilidades implícitas en los procesos de patrimonialización y etnomercantilización, como las tensiones y 
alteridades emergentes en las prácticas alimentarias de sectores estigmatizados y negados del sur global. Conceptualmente, tras haber delineado el enfoque desde el cual cobra forma este artículo, me apoyo en la perspectiva de la colonialidad del poder/saber (16) (17) (18) y en los desafíos que plantea el sociólogo portugués Boaventura de Sousa Santos, de ir al Sur y trabajar con el Sur ${ }^{(19)}$. En tanto este Sur revela un amplio pluriverso de saberes, siendo una metáfora de encuentros, confrontaciones y polinización de saberes. Entendiendo que abren una posibilidad para identificar y discutir la validez de los conocimientos y los saberes que no son reconocidos como tales por las epistemologías dominantes.

Respecto a los aspectos metodológicos, en los tres casos se emplearon técnicas que apuntaron a ajustarse a las particulares preguntas que fueron apareciendo, pero siempre basadas en las nociones de la acepción etnográfica, a través de entrevistas en profundidad y semiestructuradas, y observación participante con distintos grados de participación. Estructuralmente este artículo está dividido en tres partes. En la primera se plantean los casos, le sigue un análisis de las gastropolíticas (20) emergentes y de los elementos que encarnan categorizaciones raciales históricamente construidas. En la última, finalmente se busca mostrar conexiones entre patrimonios alimentarios emancipatorios y estrategias dentro del marco del derecho a la alimentación y los movimientos de soberanía alimentaria. A continuación, se esbozan las tres situaciones.

\section{El caso de la Quebrada, los restaurantes peruanos y las comunidades "invisibles"}

En 2003 la Quebrada de Humahuaca, perteneciente a la región noroeste de Argentina, es declarada Patrimonio Mundial por la Unesco, bajo la categoría de paisaje cultural, en un contexto de fuerte crisis económica y de expansión del turismo como una de las más dinámicas ramas de la economía internacional. En este marco, gastronomía y turismo se presentan como promotoras de la agricultura encarada por pequeños productores, a quienes se los asocia con la preservación de saberes tradicionales y productos elaborados con técnicas artesanales y en riesgo de extinción. Tras la patrimonialización se ha ido estableciendo un circuito de venta de 
DOI 10.35953/raca.v1i1.39

alimentos y comidas destinados a los turistas y viajeros. Entre estos, surgen restaurantes regenteados por pequeños empresarios que provienen de las mismas ciudades que los turistas, que se diferencian de los manejados por pobladores locales, que brindan platos regionales y minutas de conocimiento masivo en Argentina, porque ofrecen platos «de autor» a costos relativamente elevados. Por ejemplo, mientras que en los restaurantes del primer tipo el plato «diario» (conformado por una sopa y la comida del día), cuesta aproximadamente entre cinco y siete dólares, un plato en los restaurantes del segundo tipo puede rondar los veinticinco dólares. Si bien los ingredientes que utilizan son prácticamente los mismos, aparecen condimentos, formas de preparación, cocción y presentación de platos que responden a gramáticas culinarias propias de las tendencias gastronómicas «gourmet» internacionales. Podemos hallar platos con nombres que resultan extravagantes para los pobladores locales, como «crepes de kiwicha rellenos con crema de choclo", "carne de llama glaseada», etc., es decir, platos susceptibles de convertirse en mercancías que evocan «la típica» y «tradicional comida» de la Quebrada. En estos circuitos sería muy difícil hallar platos como picante de mondongo, chanfaina o sopa majada, básicamente guisados cocinados en fogones, fuertemente condimentados, mayormente elaborados por mujeres, que contienen maíz, papas criollas y cortes de carne relativamente grasosos, muchas veces provenientes de animales de las chacras. Este tipo de preparaciones suelen ser suprimidas en los menús porque son asociadas a un «escaso refinamiento» culinario y estético, a su «inadecuación» en términos nutricionales, y porque remiten al origen rural, campesino e indígena de quienes los consumen. La quinoa aparece como un alimento denigrado simbólicamente, pues quien la producía y consumía, junto con otros alimentos, era identificado bajo la tríada "pobre, indio y campesino", hasta la emergencia al menos de los procesos de fines de los '90, que buscan recuperar alimentos y técnicas de producción tradicional. Otra situación que habla de sentimientos de inadecuación, y también de ocultamiento, emerge en estas notas de campo:

Tras acompañar a un agente sanitario a la casa de un poblador que ronda los ochenta años, llamado Agapito y vive en medio de los cerros, a tres horas de Juella caminando, comentaba con él las respuestas que me dio a 
mi pregunta de "cuál era su dieta". Que para mi sorpresa había resultado algo "urbanizada": "fideos y arroz, lo que se come en todos lados" me respondió. Dentro de mi esquema de clasificaciones, suponía que la respuesta debía ser más o menos así: "maíz, carne, sopa". El agente sanitario me explicaba "él tiene miedo del rechazo, si alguien de afuera le pregunta, él va a responder lo que supone se espera que coma, incluida las verduras y todo lo que dicen los médicos. Pero que su dieta está basada en sopa con charqui, carne fresca, frangollo y harina de maíz.

Quedó claro el sentido que implica ocultar lo que se come, como si se tratase de un consumo incorrecto, vergonzoso que no remite a los cánones "modernos", y "civilizados". Y sin embargo se corresponden al tipo de preparaciones que circulan en los eventos comunitarios de la región. Las que permiten recargar energías vitales, mentales y simbólicas; son las "comidas del alma" al decir de Sidney Mintz (2), y cumplen con el requisito, de acuerdo con la voz de los interlocutores, de ser "sabrosas" y "llenar". A continuación, se retoma el caso de los inmigrantes peruanos en Buenos Aires, donde aparecen tensiones que se repiten. Sobre todo, para quienes adoptaron como lugar de residencia determinados barrios de la Ciudad de Buenos Aires (Once y Abasto), que en el imaginario de muchos porteños evoca por el origen europeo de cierta parte de su población, a "un pedacito de Europa", que remite a superioridad, civilización y progreso. En este marco los restaurantes se vislumbraron como un espacio de re-territorialización de la cultura, en donde la memoria colectiva queda inscripta como un símbolo de la identidad. De esta forma se creó una especie de frontera entre el "adentro peruano", recreado a través de una etnicidad investida de sentimientos de origen común, camaradería, vecindad y familiaridad, y el "afuera argentino". Mientras se gestaba este proceso fue publicada una nota en una revista de difusión masiva, bajo el sugerente título "La invasión silenciosa" dedicada a migrantes de países vecinos y que se extendía a Perú. En esta nota se referían a una de las comidas en particular, el cebiche. Es de señalar que este plato es uno de los "plato totem" (21) de los peruanos en el nuevo contexto, en el que se conjugan una serie de sentidos ambiguos y condensados:

Como en Perú: cebiche en las calles (...) en la zona del Abasto está el mayor asentamiento de peruanos (...) el olor penetrante a comida se siente a partir de las 11 de la mañana (...) se construyen una mesita con dos cajones de fruta, la forran con bolsas de residuo, apoyan las cacerolas oxidadas y comienzan a preparar cebiche: pescado crudo 
(...) Como los peruanos comen parados, parte de la comida cae sobre la vereda" (Revista La Primera, 4/4/00) ${ }^{(22) .}$

Según la visión de este medio, lo crudo del cebiche sería percibido por un "nosotros" civilizado como un rasgo de falta de educación e instrucción. Siguiendo a Lévi-Strauss ${ }^{(23)}$, habría una doble oposición entre elaborado y no elaborado, entre cultura y naturaleza, ya que el alimento cocinado se debe a una transformación "cultural". Curiosamente, el "sushi", de origen japonés, a pesar de ser en esencia otra variedad de pescado crudo, se visualiza como un plato sofisticado y símbolo de distinción. Basándonos en la aseveración de Harris ${ }^{(3)}$ de que los alimentos transmiten mensajes, la cocina no estaría más que reproduciendo el conflicto social: en tanto el sushi al ser japonés, y lo japonés en el imaginario colectivo occidental remite a culto y a limpio, y por lo tanto a aceptable y "bueno" en definitiva, el cebiche, en tanto se asocia a lo peruano, a lo latinoamericano, remite a la suciedad e inferioridad con que se relaciona a inmigrantes de ciertas procedencia en Buenos Aires.

Pocos años después comenzó a manifestarse otro fenómeno. Pude ir viendo que en muchos de aquellos restaurantes donde mi presencia podía llamar la atención, por blanca, por hablar de "vos" y no de "io" o "tu", dejó de hacerlo, comenzaban a ser muy frecuentes los comensales porteños y a disminuir la presencia de peruanos. Los precios poco a poco fueron encareciéndose, las porciones disminuyendo de tamaño, desapareciendo las típicas canchas (maíz tostado salado) del servicio y apareciendo paneras con pan (lo cual remite a la costumbre argentina) sin ser solicitadas. Es decir, se fueron "argentinizando". Este viraje comenzó a darse en el marco de la denominada "revolución gastronómica peruana" a nivel internacional (24). Comenzaron a aparecer en otros barrios de la ciudad elegantes y sofisticados restaurantes a cargo de empresarios "globales" (25) y cocineros especializados, cuyos platos, como, por ejemplo, "tiradito al olivo", "causa Nikkei", "salmón novoandino", "tallarines mar y tierra", remiten a gramáticas culinarias que responden a la denominada cocina de autor. Si bien en los platos hay reminiscencias a los tradicionales, estos no podrían ser comidos en ningún otro restaurante peruano. Es decir, la cocina de esta nacionalidad adquiere rentabilidad económica para un reducido número de empresarios de restaurantes de comida peruana "de autor", y de aquellos otros que lograron captar comensales mayoritariamente porteños, a través de la creación y 
DOI 10.35953/raca.v1i1.39

recreación de productos que responden a esta demanda de "producción de sensaciones" y su reutilización como nuevas manifestaciones para la recreación y el ocio. Pero el gusto por las comidas peruanas entre cierto sector del público porteño no redunda en la valorización de los usos y saberes locales que estos inmigrantes traen a cuestas y que implique modificaciones positivas en la construcción de su ciudadanía.

El último caso es el que denomino para este artículo las "comunidades invisibilizadas". En el marco de la investigación vinculada a entornos obesogénicos (26) en AMBA y zonas periurbanas de Buenos Aires donde habitan poblaciones de raíces y origen paraguayo volvemos a encontrar una continuidad de elaboraciones culinarias propias de los lugares de procedencia de estos migrantes. En uno de los barrios populares más grandes de la Ciudad de Buenos Aires, conocido como Villa 21-24 (en adelante llamado barrio), aparece la presencia y continuidad de ciertos saberes familiares y platos regionales como chipa, sopa paraguaya, etc. Si bien estas preparaciones fueron mencionadas como esporádicas debido a los costos de los ingredientes por parte de las interlocutoras, el acceso a los mismos, como distintos tipos de maíz, mandioca, harinas, legumbres, queso y condimentos, propios de muchas zonas de Paraguay, está presente. En nuestras recorridas por el barrio observamos un amplio circuito de pequeños comercios dispersos en las angostas calles y pasillos que ofrecen harina de maíz amarillo recién molida, quesos del tipo de los que se acostumbra a utilizar en Paraguay para hacer sopa paraguaya, chipá guazú, etc. En las verdulerías del barrio suele destacarse la exhibición de mandioca entre la oferta de las demás verduras y frutas. También es frecuente ver una especie particular de porotos marrones y pequeño, que además pueden hallarse en el resto de los puestos de las calles centrales del barrio, junto a la sopa paraguaya, carne a la parrilla con mandioca hervida, etc.

Por otro lado, la zona periurbana donde realizamos el trabajo de campo en el marco de esta investigación se ubica a $70 \mathrm{~km}$, aproximadamente, de la Ciudad de Buenos Aires. La práctica totalidad de nuestros interlocutores son de origen boliviano y se dedican a la producción de verduras para su venta. Lo que se desprende es nuevamente, la existencia de prácticas de preparación y consumo de alimentos regionales, como humitas, tamales, guisos sopas, etc., que, si bien no están dentro de 
DOI 10.35953/raca.v1i1.39

los repertorios cotidianos, su consumo suele estar presente en reuniones comunitarias, fines de semana, festejos, épocas estivales cuando es posible disponer de los ingredientes. Pudimos observar también que en pequeños sectores de los predios suelen plantarse algunas variedades de maíz, papas, ajíes, incluso quinoa y amaranto. Esto es posible porque quienes viajan a su tierra de origen traen semillas de estos alimentos, que, al cabo de ciertos años, logran adaptar a las condiciones climáticas de Buenos Aires. En estos dos contextos, uno urbano y otro periurbano, en los que se hace evidente la continuidad de prácticas alimentarias de las zonas de origen, hallamos en las entrevistas apreciaciones sobre dichos consumos como los siguientes:

está el bori, las albóndigas grandes, con queso, son pesadas, una bomba (...) el paraguayo no usa mucho puré (enva(Vecina del barrio 21/24, 40 años, inmigrante).

plantamos cuatro surcos para el consumo (...) y cuando están tiernos hacemos humitas y tamales (...) molemos y ahí nos juntamos todas las mujeres de la familia y empezamos a hacer entre todas, (...) lo pelamos con ceniza, (...) y es como amasar el pan, y se va pelando (...) después lo molemos en la máquina que es a mano y para el recado compramos dos cabezas de chancho (...) pero a los chicos (se refiere a sus hijos de 3 y 6 años) les doy poquito porque es pesado.

Entre las preparaciones caseras son notorias las propias de los contextos de procedencia de migrantes, con ingredientes naturales o mínimamente procesados ya mencionados. Llama la atención la valoración ambigua por parte de las entrevistadas respecto a estas comidas, pues mientras notamos el entusiasmo y conocimiento pormenorizado para llevar adelante distintas preparaciones, siendo incluso partícipes de situaciones en las que se preparan estas comidas, contextos de reunión, sociabilidad y clima distendido, aparecen recurrentemente valoraciones negativas, vinculadas a lo pesado, como grasoso de las comidas.

\section{Lo que queda por debajo del mantel ¿de qué nos hablan estas situaciones}

En lo que sigue examinaré algunos aspectos que emergen de los casos presentados y que reflejan paradojas de las prácticas alimentarias y su patrimonialización. El caso que aparece con mayor potencia para analizar las tensiones que encarna el 
imperativo de la salvaguardia de la patrimonialización por Unesco, ciñéndonos al campo de lo alimentario, es el de la Quebrada. Sabemos que la alimentación entre otras dimensiones está asociada a la historia, al conocimiento, la identidad, la memoria colectiva y el ambiente También que el auge del patrimonio está ligado con procesos de democratización de la sociedad y con los principios de los derechos humanos, la equidad, la sostenibilidad, y el respeto mutuo entre comunidades ${ }^{(4)}$. Sin embargo, resulta llamativo que el propósito de reconocer y proteger los conocimientos, prácticas y productos alimenticios de las cocinas regionales como factores fundamentales de la identidad, pertenencia y bienestar de la población se confronta con otra situación. En el caso que se presenta emerge la percepción de que los saberes y los productos configurados en el ecosistema local, las historias de ocupación territorial, entendidas como pilar de la identidad, son percibidas como simples, pobres, con poco refinamiento culinario y estético, e inadecuadas en términos nutricionales. Pues mientras hacia el sector turístico se exalta la diversidad del pasado y se resalta el origen rural, campesino, indígena de la cocina, se marca la falta de higiene y el poco refinamiento culinario. Lo que subyace es que los saberes de las poblaciones vinculadas con dicha diversidad culinaria, (la que se busca resguardar) no son producidos de acuerdo con las metodologías aceptadas. O porque son producidos, desde la perspectiva decolonial, por sujetos "ausentes", considerados incapaces de producir conocimientos válidos debido a su condición o naturaleza subhumana.

Esta categorización que leemos como racializada tiene una historia de larga duración y se reitera desde distintos ámbitos del poder del norte global. Desde el periodo colonial, muchas de las tensiones sociales se manifestaron en las clasificaciones y jerarquizaciones que los conquistadores hicieron de las cocinas, las dietas y los productos nativos, de acuerdo con su propia experiencia cultural del alimento y de su percepción del mundo indígena. Así, algunos de los alimentos fueron concebidos como sucios, feos, inferiores e incivilizados (27) (28). En efecto, la colonización de millones de personas logro inculcar sabiamente el complejo de inferioridad, el temor. Con múltiples matices procuró, a través de las distintas instituciones modernas como la escuela, el sector salud, el trabajo, marcar a fuego las virtudes civilizatorias eurocéntricas, en busca de reducir al otro colonizado a un ser inferior que habita una zona del "no ser" (29). Esto significó la marginación social y productiva de ciertos productos tradicionales o su erradicación, como 
DOI 10.35953/raca.v1i1.39

sucedió con la quinua y el amaranto, y su reemplazo por especies europeas. La jerarquización de los alimentos actuó como un mecanismo efectivo para trazar diferencias y distancias sociales entre aquellos productos o comidas que se consideraban naturalmente destinados para las castas más elevadas y los naturalmente aptos para las castas subalternas ${ }^{(27)}$.

Por otro lado, las intervenciones y las políticas estatales modernas han incidido en la transformación de los "malos hábitos" alimentarios de estos pueblos mediante el cambio cultural dirigido y de acuerdo con nociones hegemónicas de progreso, modernidad, higiene y salud (30) (31) (27). Por esta vía la colonización epistémica logró disciplinar, adecuar, civilizar las mentes de los colonizados a las demandas económicas, sociales y culturales del capitalismo industrial moderno y a su agresiva demanda de inversiones de mercado y fuentes de materia prima ${ }^{(28)}$. Proyecto civilizatorio del cual las distintas élites regionales, que comparten las mismas aspiraciones del norte global, han buscado distanciarse emulando dietas y modales extranjeros ${ }^{(27)}$. No en vano, tanto en Quebrada como en el periurbano bonaerense, mis interlocutores han comentado en diferentes situaciones que "la pala" (para trabajar la tierra y producir alimentos) es vista como el último recurso en el proyecto de vida para sus hijos/as, pues subyace que el trabajo en la tierra es para "brutos" y "negros". Desde la perspectiva decolonial del poder esta situación apunta a pensar que las políticas de la Unesco parecen quedar atrapadas en una agenda de expansión cultural global neoliberal sustentada en ejes discursivos civilizatorios, que reproduce subalternidades culturales a nivel global, dada la inercia del organismo internacional para desmadejar los discursos eurocéntricos que se depositan en estas omisiones ${ }^{(32)}$. Si el patrimonio inmaterial de la humanidad suponía un avance para la inclusión de patrimonios culturales silenciados, tras los años transcurridos parecen instaurar a través de su potencialidad para vehiculizar narrativas e ideales eurocéntricos, un marco de expansión de esta fase del neoliberalismo.

\section{La riqueza culinaria de las comunidades, lo que hace el capital global}

La lógica mercantilista encarnada en las políticas de patrimonialización y etnomercantilización de los eventos alimentarios, empeñada en la espectacularización de la diversidad y riqueza culinaria de las comunidades obsturan la posibilidad de mostrar la 
conflictiva historia cultural, económica y colonial subyacente. Nos referimos a los efectos de las políticas macroeconómicas y agroalimentarias sobre los sectores campesinos productores de alimentos y la intricada situación alimentaria y nutricional a nivel internaciónal. Es de destacar que la inseguridad alimentaria afecta a 187 millones de personas en América Latina y el Caribe. Paralelamente, por cada persona que sufre hambre, más de seis sufren sobrepeso u obesidad (33). Las propuestas de patrimonialización gastronómica suelen convivir acríticamente en el mismo plano de la expansión de los mercados de las grandes corporaciones en sus diferentes actividades agroalimentarias, naturalizando el lugar que ocupa el capital financiero en la expoliación cultural, social, política, de los sures globales.

Las grandes empresas del sector de la industria alimentaria al ampliar los espacios comerciales de sus productos moldean los hábitos alimentarios a través del bombardeo publicitario y el llamado "secuestro de paladar" (34) orientados al consumo excesivo de productos ultraprocesados. Lo cual es adoptado por consumidores tanto en los países desarrollados como en los más pobres. Pensemos en la agresividad comercial implícita que se observa en el supermercado flotante de la empresa Nestlé, navegando por los igarapés de la Amazonía para llegar a la población ribereña aún lejana de los hábitos de consumo prevalente en los grandes centros urbanos (1). El ejemplo nos permite ver el poder del capital global en su empeño por alcanzar las regiones más pobres y en el intento por sustituir los productos locales por los de la agroindustria globalizada. A pesar de la independencia de los países, esta forma de colonialismo, que es la manera en que se expresa el capitalismo en su faceta neoextrativsta de la actualidad, no puede ser vista como pasado.

Claramente podemos ver que persiste como estructura histórica, ya que incide en la restricción de la diversidad biológica, poniendo en peligro el modelo alimentario existente. La aparición de estos productos modifica la forma en que las personas interactúan con los alimentos "tradicionales", incidiendo en su abandono, cada vez menos al alcance de los sectores más pobres. Repercutiendo en los cambios en el patrón alimentarios y tipo de habilidades culinarias necesarias para la elaboración de comidas ${ }^{(35)}$. Se trata no solo de la perdida de saberes, de la riqueza de cultural, también nutricional, pues en términos generales se trata de elaboraciones que coinciden notablemente con los principios actuales de la alimentación sana. Que implican, en general, el consumo de granos 


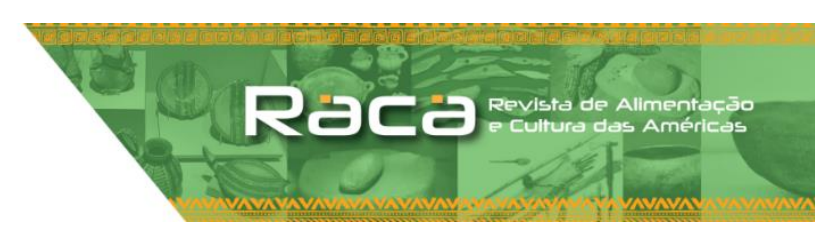

DOI 10.35953/raca.v1i1.39

integrales, de fibras, variedad de verduras, la ingestión moderada de productos de origen animal, azúcar en su forma original e incorporación de micronutrientes. Si los proyectos de salvaguardia de las tradiciones alimentarias locales realmente buscan abogar por su rescate, una manera de hacerlo podría ser poniendo énfasis en la visibilización de esta situación, como también habilitando la denuncia. Pues de lo contrario los proyectos de ampliación de los mercados de las grandes empresas de abastecimiento y fast food, que inciden en la desaparición de productos y expoliación de saberes, seguidos por proyectos empeñados en "salvar" la gran riqueza en riesgo de extinción parecen mantener límites borrosos. En Quebrada advertimos que las comidas frecuentes están en mayor medida constituidas por preparaciones cuyos ingredientes principales provienen del mercado. El caso más significativo es el de la Coca Cola que muestra el poder de las grandes corporaciones de la industria alimentaria a escala global y la fuerte publicidad, en pos de la creación de significados simbólicos que instan a su consumo y organizan nuevas prácticas entre la gente de la zona, como la de ofrecerla a la Pachamama, en tanto expresión de estatus y amor hacia ella. Aunque la bebida verdaderamente autóctona sea probablemente la chicha, prohibida su venta en la zona desde la segunda parte del siglo $\mathrm{XX}$, debido a sus dudosas cualidades bromatológica. Pero que sin embargo muchas mujeres adultas continúan elaborando "puertas adentro" y no para el turismo.

En el caso de las "comunidades invisibilizadas", la de los barrios populares del conurbano y periurbano bonaerense, vemos que, aunque las personas que han migrado modifican su alimentación de acuerdo con sus ingresos, y el nuevo entorno les induce a consumir alimentos de las grandes industrias, existen prácticas de resistencia para conservar el "sabor de su tierra" en los nuevos contextos. Las mujeres a través de sus trabajos juegan un importante papel cultural en la formación de tradiciones y gustos alimentarios regionales, quienes haciendo uso de sus conocimientos dejan su impronta culinaria en las ventas callejeras. Sin embargo, estas formas culinarias esto no genera interés por parte de proyectos de patrimonialización para su valorización económica y cultural, como abrir el camino al reconocimiento de las comunidades migrantes. Nos preguntamos si sería posible impulsar el desarrollo del turismo gastronómico en los barrios populares y periurbanos bonaerenses donde realizamos nuestro estudio, a los fines de contribuir a la creación de oportunidades, puestos de trabajo y permitir a las comunidades reforzar sus identidades, preservar sus recetas, enaltecer su capital cultural 
y el orgullo de la comunidad. Por el momento sabemos que propuestas de este tipo quedan al resguardo del capital financiero. La conexión con corrientes migratorias de países limítrofes de fuerte presencia en Argentina no las sitúa como campo de interés para aquellos actores con capacidad para "otorgar" mayor valor agregado y ocuparse de la mercantilización de la diversidad natural y cultural y en su patrimonialización. El proyecto por parte del capital para estos contextos pareciera ser el de impulsar inversiones que incrementen el mercado de los productos ultraprocesados "buenos para vender" y expansión de cadenas de fast food.

Posiblemente proyectos de patrimonialización "popular" tampoco generen demasiado interés para los actores de las comunidades en cuestión. En primer lugar, porque las habilidades y conocimientos culinarios suelen estar naturalizados, constituyen un bien colectivo y no suelen pensarse como recurso comercial más allá del que puede tener para los compatriotras, quienes al consumir "sus comidas" encuentran una manera de conectarse con su tierra de origen. En segundo lugar, porque las huellas de la colonización epistémica son profundas. Nos parece ver que la ideología eurocéntrica que incide en el desprecio de las culturas que remiten al Sur Global está internacionalizada. Reminiscencias que hablan de la incapacidad para reconocerse como sujetos con saberes y experiencias las encontramos en las recurrentes apreciaciones sobre la pesadez de las elaboraciones tradicionales que los migrantes traen consigo. En esta dirección hay una situación que vuelvo a traer, la del consumo del pescado "crudo" entre los migrantes peruanos. Su consumo era asociado a las condiciones de pobreza y suciedad en que era preparado. Reforzando una imagen que muestra a la cultura peruana como atrasada, inestable, incierta, bárbara; en síntesis, diferente, reprobable y rechazable. Por ello, en el cebiche, al menos para la sensibilidad de un sector de la sociedad receptora, quedaron plasmadas las marcas xenófobas y discriminatorias.

\section{Conclusiones}

En la búsqueda por mostrar algunos ejes que sustentan la selección de lo que se decide visibilizar e invisibilizar a la hora de patrimonializar y producir etnomerancias, quedan expuestas las dinámicas conformadas en la esfera internacional. Estas son las que delinean las referencias de producción y consumo alimentario y sustentan la 
redefinición de culturas y tradiciones alimentarias en las sociedades locales. El campo de la patrimonialización queda sujeto a esta lógica, donde el mercado adquiere un peso contundente en la definición de los patrimonios y en el tratamiento de la otredad, convertidos en un recurso comercial. Esta lógica está anclada a la reproducción de la supremacía blanca y las jerarquías de conocimiento que se encarnan en los prejuicios sociales. Estos provocan legados alimentarios "otros" que no parecen despertar interés por parte del mercado para su promoción. Que a su vez legitiman las relaciones de superioridad/inferioridad, reproduciendo legados coloniales que perpetúan la desigualdad social.

En este sentido encontramos que la perspectiva decolonial muestra su potencial para el análisis antropológico de las visibilidades e invisibilidades del patrimonio A través de los tres casos presentados se ha intentado mostrar las marcas en que quedan plasmadas la creación de la alteridad como un espacio vacío de saberes. En los de la Quebrada y en el de los peruanos tras el boom gastronómico, aparecen los alimentos, las preparaciones, los saberes, que remiten a la otredad que hay que "limpiar" en el escenario de los circuitos gourmetizados. En el caso de los inmigrantes de barrios populares y productores de verduras del periurbano, la otredad alimentaria es rechazada e invisibilizada en el seno de la sociedad receptora. En todos los casos los alimentos, elaboraciones y comidas remiten a sistemas de clasificación a través de los cuales se construye la naturaleza, la cultura y la sociedad, diferente a las formas modernas.

Pero mientras en los dos primeros casos se constituyen, tras la racialización de las recetas, en frentes de negocio de comida típica en una "ficción gourmet", en el segundo las recetas siguen conformando parte del patrimonio colectivo, vivido y real. Todo esto se traduce en la importancia de pensar en políticas patrimoniales que conecten a quienes producen los alimentos con quienes los preparan, regulando y defendiendo la autonomía de las comunidades. Cuidando de no reproducir la explotación e invisibilización de las mujeres, responsables ocultas en general de la actualización de las preparaciones y continuidades de legados culinarios. Generado herramientas para deconstruir, desmantelar y descolonizar las prácticas y sentidos sociales sedimentados sobre una visión colonial y eurocéntrica. Abriendo paso a la identificación y validación de conocimientos que pueden reinventar la emancipación social y la liberación. Por esta via habilitar a pensar en propuestas que se fundamenten en el derecho a la alimentación. Lo 
cual, nuevamente, requiere romper con jerarquías racializadas e imaginar la comida como parte del colectivo, la comunidad. Remarcando la necesidad de relaciones sociales de solidaridad con la naturaleza y el sostenimiento de la salud y la biodiversdiad. Esto incluiría el sostenimiento de propuestas de seguridad, soberanía y autonomía alimentaria de comunidades alterizadas. Es el desafío de los proyectos culinarios emancipatorios.

\section{Referencias}

1. Paula, Maciel de. Evolucion del Sistema Agroalimentario Mundial. Curitiba : CRV, 2017.

2. Mintz. Sabor a comida, sabor a libertad. Mexico : Ediciones de la reina roja, 2003.

3. Sammartino, G. Notas para identificar el modelo de producción. Buenos Aires : Diaeta, 2014, Vol. 32 HYPERLINK "http://www.aadynd.org.ar/descargas/diaeta/02Sanmartino.pdf" http://www.aadynd.org.ar/descargas/diaeta/02-Sanmartino.pdf.

4. Marvin, Harris. Bueno para comer . Madrid : Alianza, 1999.

5. Unicef. Convención de Salvaguardia del Patrimonio Cultural. Unesco, 2003 Consultado el 12 de febrero de 2016.

unesdoc.unesco.org/images/0013/001325/132540s.pdf.

6. Prats, L. La mercantilización del patrimonio: entre la economía turística y las representaciones identitarias. . s.I. : PH Boletín del Instituto andaluz del Patrimonio Histórico, 2006, Vols. 58, 72-80. HYPERLINK "http://www.iaph.es/revistaph/index.php/revistaph/article/view/2176" http://www.iaph.es/revistaph/index.php/revistaph/article/view/2176 .

7. Kirshenblatt-Gimblett, B «La cultura de les destinacions: Teoritzar el patrimoni».. s.I. : Revista d'Etnologia de Catalunya, , 2001, Vols. 19, 44-61. HYPERLINK "file:///C:IUsers||DELLI|Downloads||49244-Text\%20de\%20l'article-60821-1-1020070103\%20(6).pdf" file:///C:/Users/DELL/Downloads/49244-Text\%20de\%20l'article$\underline{60821-1-10-20070103 \% 20(6) . p d f}$

8. Boltanski, L. y Chiapello, E El nuevo espíritu del capitalismo.. Madrid : Akal, 2002.

9. Serra, J., Pascual, M. Patrimonio.alimentario,turismo.y.espectáculo .Reflexiones.en.torno..

a.un.proyecto.de.desarrollo.de.experiencias.turísticas.gastronómicas Joan Ribas Serra, Margalida Mulet Pascual . Polisemias de la Alimentación. Salud, desperdicio, hambre y patrimonio. Barcelona : Universitat de Barcelona, 2019 HYPERLINK "https://www.researchgate.net/profile/Cristina_Larrea_Killinger/publication/331529848_La s_relaciones_entre_salud_y_alimentacion_Una_lectura_antropologica/links/5c7e7a8545 $8515831 \mathrm{f} 855 \mathrm{~b} 4 \mathrm{f} /$ Las-relaciones-entre-salud-y-alimentacion-Una-lectura- 
antropologica.pdf" V| "page=77"

https://www.researchgate.net/profile/Cristina Larrea Killinger/publication/331529848 La $\mathrm{s}$ relaciones entre salud y alimentacion Una lectura antropologica/links/5c7e7a8545 8515831f855b4f/Las-relaciones-entre-salud-y-alimentacion-Una-lecturaantropologica.pdf\#page $=77$.

10. P, Menesses. Colonialismo como violência: a "missão civilizadora" de Portugal em Moçambique. 115-140, s.l. : Revista Crítica de Ciências Sociais, 2018, Vols. número especial, novembro HYPERLINK "https://journals.openedition.org/rccs/7741" https://journals.openedition.org/rccs/7741.

\section{Comaroff J, Comaroff J. Etnicidad S.A. . Madrid : Katz Editores, 2011.}

12. Bornand, I. Nação e alteridade no âmbito da alimentação: apontamentos sobre o caso chileno. Revista de Alimentação e Cultura das Américas. 2019, Vol. 1, 1 HYPERLINK "file:///C:IUSsers||DELLI|Downloads|\4-Article\%20Text-30-1-1020190130.pdf" file:///C:/Users/DELL/Downloads/4-Article\%20Text-30-1-10-20190130.pdf

13. G., Sammartino. ¿Por qué seguimos comiendo los alimentos de la chacra?. TESSITURAS: Revista de Antropología e Arqueología. 2, 2015, Vol. 3. HYPERLINK "https://periodicos.ufpel.edu.br/ojs2/index.php/tessituras/article/view/6050" https://periodicos.ufpel.edu.br/ojs2/index.php/tessituras/article/view/6050

14. - . "Peruvians restaurants in Buenos Aires (1999 - 2009) From warm (coll) self segregation to otherness commodification" . Anthropology of food, 2010, Disponible en http://aof.revues.org.

15. Sammartino G, Benza S. En el interior de los restaurantes, las señas de la peruanidad. [aut. libro] Medina X. Alimentación y Migraciones en Iberoamérica. España : UOC, 2014.

16. Quijano A.Coloniality of Power, Eurocentrism, and Latin America. 3, s.I. : Nepantla: Views from South, 2000, Vol. 1. 533 -580. HYPERLINK "https://journals.sagepub.com/doi/abs/10.1177/0268580900015002005" https://journals.sagepub.com/doi/abs/10.1177/0268580900015002005

17. Lander, E. (comp). La colonialidad del saber: eurocentrismo y ciencias sociales perspectivas latinoamericanas. Buenos Aires : CLACSO, 2000.

18. Mignolo, W. Historias Locales/diseños globales. Colonialidad, conocimientos subalternos y pensamiento fronterizo. Madrid : Akal, 2003.

19. Santos, B. Epistemologies of the South and the Future". From the European South, 2016, Vol. 1. 17-19.

20. Appadurai, A. Gastro-politics in Hindu South Asia. American Ethnologist, 1981, Vol. 8. 494-511. HYPERLINK 
"https://anthrosource.onlinelibrary.wiley.com/doi/pdf/10.1525/ae.1981.8.3.02a00050" https://anthrosource.onlinelibrary.wiley.com/doi/pdf/10.1525/ae.1981.8.3.02a00050 .

21. Fischler, C. El (h) omnívoro. El gusto, la cocina y el cuerpo. Barcelona : Anagrama, 1995.

22. La Primera de la Semana, Año 1, No 3, 4 de abril de 2000. La invasion silenciosa. Buenos Aires : s.n., 2000. 3, 4 de abril.

23. Lévy-Straus, C. Lo crudo y lo cocido. Mexico : Fondo de Cultura Economica, 1965.

24. Lauer, M y Lauer, V. la revolución gastronómica peruana. Lima : Universidad de San Martín de Porres, Facultad de Ciencias de la Comunicación, Turismo y Psicología., 2006.

25. Imilan, W. Restaurantes peruanos en Santiago de Chile: construcción de un paisaje de la migración.. s.I. : Revista de Estudios Sociales, 2014, Vol. 48. 15-28. HYPERLINK "https://revistas.uniandes.edu.co/doi/pdf/10.7440/res48.2014.02"

https://revistas.uniandes.edu.co/doi/pdf/10.7440/res48.2014.02

26. Swinburn B, Egger G, Raza F. Dissecting obesogenic environments: the development and application of a framework for identifying and prioritizing environmental interventions for obesity.. s.I. : Preventive Medicine, 1999, Vol. 29. 563-570.

HYPERLINK "https://publichealthathens.com/wp/wp-content/uploads/simple-filelist/Dissecting_Obesogenic_Environments_The_D.pdf"

https://publichealthathens.com/wp/wp-content/uploads/simple-file-

list/Dissecting Obesogenic Environments The D.pdf

27. Saldarriaga, G. 2009. "Comedores de porquerías: control y sanción de la alimentación indigena, desde la óptica española en el Nuevo Reino de Granada (siglos XVI y XVII)". 2, s.I. : Revista de Historia Iberoamericana, 2009, Vol. 2. 16-37.

HYPERLINK "file:///C:IUUsers||DELLIIDownloads||Dialnet-ComedoresDePorquerias3621061\%20(1).pdf" file:///C:/Users/DELL/Downloads/Dialnet-ComedoresDePorquerias$\underline{3621061 \% 20(1) . p d f}$

28. Camacho, J Una cocina exprés. Cómo se cocina una política pública de patrimonio culinario. .. s.l. : El valor del patrimonio: mercado, políticas culturales y agenciamientos sociales, 2014. 169-200.

https://d1watxts1xzle7.cloudfront.net/38273631/Como se cocina una politica de patri monio.pdf?1437673994=\&response-content-

disposition=inline\%3B+filename\%3DUna cocina expres. Como se cocina una po.pdf \&Expires=1591127776\&Signature=likgeOnL2D5xIW9hQWmn IDzaKBelwi2q0ud h0vTT bDKrQBsowr9gfWrOfhZ6VzYsAHW0ljhzsIK0T7Tbjy6dsasoECNAtY3UiEvFNb9okLydZ0i OgY1M1zIm2VX8aiQGcLrLzRomuB7FtDBv-8vmL9x -9-8u 7IRYNhg9ry6sCwNG-

FCMJiXuOVnw92mmGmi5ggHC6cvtYsioJFyq7A tqtnfuwxkBKIFONfxGzKUiZK 1meLdY It|65gAqEUCbG1txH3MdG2plllZw-IF4HbLAJDB6vrX3ieJCLsrVLz-

UZz5W fQmo17aLFU L4WHeOIHT-cHmabloE8ceCAQ \&Key-Pair-

Id=APKAJLOHF5GGSLRBV4Z 
29. Fanon, Frantz (1952), Peau noire, masques blancs. Paris: Éditions du Seuil.

30. Escobar, A. Encountering Development: The Making and Unmaking of the Third World: Princeton: Princeton University Press, 1995. HYPERLINK

"https://books.google.es/books?hl=es\&lr=\&id=Z887lsgjLuwC\&oi=fnd\&pg=PP1\&dq=Escob ar,+A.+Encountering+Development:+The+Making+and+Unmaking+of+the+Third+World: +Princeton:+Princeton+University+Press, +1995.\&ots=bnm4q7u_5i\&sig=0WXlyfCoBiRYTfGkN-Ze5DLG3I" \

"v=onepage\&q=Escobar\%2C\%20A.\%20Encountering\%20Development\%3A\%20The\%20 Making\%20and\%20Unmaking\%20of\%20the\%20Third\%20World\%3A\%20Princeton\%3A \%20Princeton\%20University\%20Press\%2C\%201995.\&f=false" https://books.google.es/books?hl=es\&lr=\&id=Z887lsgiLuwC\&oi=fnd\&pg=PP1\&dq=Escob ar, +A.+Encountering+Development:+The+Making+and+Unmaking+of+the+Third+World: +Princeton:+Princeton+University+Press, +1995 .\&ots=bnm4q7u 5i\&sig=0WXlyfCoBiRYTfGkN-

Ze5DLG3|\#v=onepage\&q=Escobar\%2C\%20A.\%20Encountering\%20Development\%3A\% 20The\%20Making\%20and\%20Unmaking\%20 of\%20the\%20Third\%20World\%3A\%20Prin ceton\%3A\%20Princeton\%20University\%20Press\%2C\%201995.\&f=false

31. Escobar, A. "El lugar de la naturaleza y la naturaleza del lugar: ¿globalización o posdesarrollo?". [aut. libro] E. Lander. La colonialidad del saber: eurocentrismo. Buenos Aires: : Clacso y Unesco., 2000.

32. Madroñal, A. y Hernández, E.Las politicas patrimoniales de la Unesco en la geopolítica de colonialidades globales y la emergencia de nuevos sentidos de interculturalidad del patrimonio en Andalucia". Revista OPSIS, 16(1): 131-152. Doi: https://doi.org/10.5216/o.v16i1.37021.

33. FAO, FIDA, UNICEF, PMA, OMS. El estado de la seguridad alimentaria y la nutrición en el mundo Fomentando la resiliencia climática en aras de la seguridad alimentaria y la nutrición. Roma: FAO, 2018 [citado marzo 17, 2019]. Disponible en:

http://www.fao.org/3/i9553es/i9553es.pdf

34. Colindres, A y Sosa López, V Migrar para sobrevivir: Observatorio del Derecho a la Alimentación y Nutrición., 2019, Vol. 11. 54-61 HYPERLINK "https://www.righttofoodandnutrition.org/files/rtfn-watch11-2019_esp-54-61.pdf" https://www.righttofoodandnutrition.org/files/rtfn-watch11-2019 esp-54-61.pdf

35. Ripe, C . Goodbye culinary cringe. Sydney: Allen \& Unwin., 1996. 